CRYSTALLOGRAPHIC COMMUNICATIONS

ISSN 2056-9890

\section{Crystal structure of cyclosulfamuron}

\author{
Gihaeng Kang, Jineun Kim,* Eunjin Kwon and Tae Ho \\ Kim*
}

Department of Chemistry and Research Institute of Natural Sciences, Gyeongsang National University, Jinju 660-701, Republic of Korea. *Correspondence e-mail:

thkim@gnu.ac.kr, jekim@gnu.ac.kr

Received 25 July 2015; accepted 27 July 2015

Edited by W. T. A. Harrison, University of Aberdeen, Scotland

The title compound (systematic name: 1-\{[2-(cyclopropylcarbonyl)anilino]sulfonyl\}-3-(4,6-dimethoxypyrimidin-2-yl)urea), $\mathrm{C}_{17} \mathrm{H}_{19} \mathrm{~N}_{5} \mathrm{O}_{6} \mathrm{~S}$, is a pyrimidinylsulfonylurea herbicide. The dihedral angles between the mean planes of the central benzene ring and the cyclopropyl and pyrimidinyl rings are 75.32 (9) and $88.79(4)^{\circ}$, respectively. The $\mathrm{C}$ atoms of the methoxy groups lie almost in the plane of the pyrimidine ring [deviations $=0.043(2)$ and $0.028(2) \AA$ ] and intramolecular $\mathrm{N}-\mathrm{H} \cdots \mathrm{N}, \mathrm{N}-\mathrm{H} \cdots \mathrm{O}$ and $\mathrm{C}-\mathrm{H} \cdots \mathrm{O}$ hydrogen bonds all close $S(6)$ rings. In the crystal, $\mathrm{N}-\mathrm{H} \cdots \mathrm{O}$ and $\mathrm{C}-\mathrm{H} \cdots \mathrm{O}$ hydrogen bonds and weak $\pi-\pi$ interactions [centroidcentroid distances $=3.6175$ (9) and 3.7068 (9) $\AA$ ] link adjacent molecules, forming a three-dimensional network.

Keywords: crystal structure; hydrogen bonding; $\pi-\pi$ interactions.

CCDC reference: 1415211

\section{Related literature}

For information on the herbicidal properties of the title compound, see: Sarıül \& İnam (2009). For a related crystal structure, see: Xia et al. (2008).

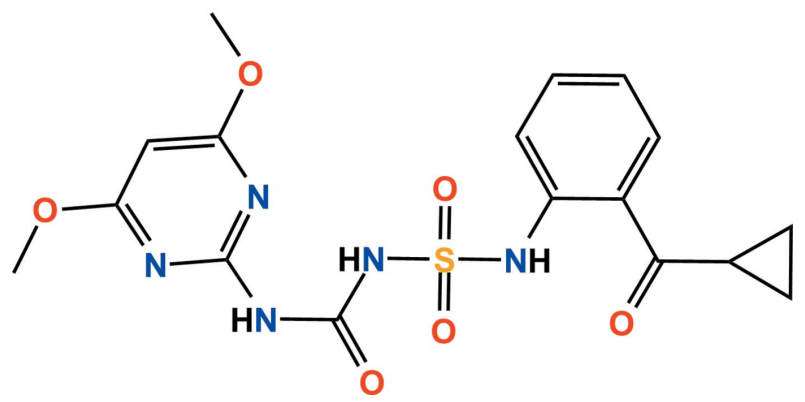

\section{Experimental}

2.1. Crystal data

$\mathrm{C}_{17} \mathrm{H}_{19} \mathrm{~N}_{5} \mathrm{O}_{6} \mathrm{~S}$

$M_{r}=421.43$

Monoclinic, $P 2_{1} / n$

$a=12.7019$ (4) $\AA$

$b=9.6216(3) \AA$

$c=15.6213(5) \AA$

$\beta=93.6194(12)^{\circ}$

$V=1905.31(10) \AA^{3}$

$Z=4$

Mo $K \alpha$ radiation

$\mu=0.22 \mathrm{~mm}^{-1}$

$T=173 \mathrm{~K}$

$0.32 \times 0.27 \times 0.23 \mathrm{~mm}$

\subsection{Data collection}

Bruker APEXII CCD diffractometer

Absorption correction: multi-scan (SADABS; Bruker, 2013)

$T_{\min }=0.934, T_{\max }=0.952$

17544 measured reflections 4365 independent reflections 3688 reflections with $I>2 \sigma(I)$ $R_{\text {int }}=0.030$

\subsection{Refinement}

$R\left[F^{2}>2 \sigma\left(F^{2}\right)\right]=0.039$

$w R\left(F^{2}\right)=0.113$

$S=1.05$

4365 reflections

264 parameters

$\mathrm{H}$-atom parameters constrained

$\Delta \rho_{\max }=0.22{\mathrm{e} \AA^{-3}}^{-3}$

$\Delta \rho_{\min }=-0.49 \mathrm{e} \AA^{-3}$

Table 1

Hydrogen-bond geometry $\left(\AA,^{\circ}\right)$.

\begin{tabular}{|c|c|c|c|c|}
\hline$D-\mathrm{H} \cdots A$ & $D-\mathrm{H}$ & $\mathrm{H} \cdots A$ & $D \cdots A$ & $D-\mathrm{H} \cdots A$ \\
\hline $\mathrm{N} 1-\mathrm{H} 1 N \cdots \mathrm{O} 1$ & 0.88 & 1.86 & $2.5736(18)$ & 137 \\
\hline $\mathrm{N} 2-\mathrm{H} 2 N \cdots \mathrm{N} 4$ & 0.88 & 1.92 & $2.6158(18)$ & 135 \\
\hline $\mathrm{C} 9-\mathrm{H} 9 \ldots \mathrm{O} 3$ & 0.95 & 2.45 & $3.088(2)$ & 124 \\
\hline $\mathrm{N} 3-\mathrm{H} 3 N \cdots \mathrm{O} 2^{\mathrm{i}}$ & 0.88 & 2.08 & $2.9391(17)$ & 165 \\
\hline $\mathrm{C} 2-\mathrm{H} 2 B \cdots \mathrm{O} 2^{\mathrm{ii}}$ & 0.99 & 2.51 & $3.483(2)$ & 169 \\
\hline $\mathrm{C} 3-\mathrm{H} 3 \cdots \mathrm{O} 4^{\mathrm{iii}}$ & 1.00 & 2.51 & $3.286(2)$ & 135 \\
\hline $\mathrm{C} 8-\mathrm{H} 8 \cdots \mathrm{O}^{\text {iv }}$ & 0.95 & 2.50 & $3.307(2)$ & 142 \\
\hline
\end{tabular}

Symmetry codes: (i) $-x+\frac{5}{2}, y-\frac{1}{2},-z+\frac{1}{2}$; (ii) $-x+2,-y+1,-z$; (iii) $-x+2,-y,-z$; (iv) $-x+\frac{3}{2}, y-\frac{1}{2},-z+\frac{1}{2}$.

Data collection: APEX2 (Bruker, 2013); cell refinement: SAINT (Bruker, 2013); data reduction: $S A I N T$; $\operatorname{program}(\mathrm{s})$ used to solve structure: SHELXS97 (Sheldrick, 2008); program(s) used to refine structure: SHELXL2013 (Sheldrick, 2015); molecular graphics: DIAMOND (Brandenburg, 2010); software used to prepare material for publication: SHELXTL (Sheldrick, 2008).

\section{Acknowledgements}

This research was supported by the Basic Science Research Program through the National Research Foundation of Korea (NRF) funded by the Ministry of Education, Science and Technology (No. 2015R1D1A4A01020317).

Supporting information for this paper is available from the IUCr electronic archives (Reference: HB7470). 


\section{data reports}

\section{References}

Brandenburg, K. (2010). DIAMOND. Crystal Impact GbR, Bonn, Germany. Bruker (2013). APEX2, SAINT and SADABS. Bruker AXS Inc., Madison, Wisconsin, USA.
Sarıgül, T. \& İnam, R. (2009). Electrochim. Acta, 54, 5376-5380.

Sheldrick, G. M. (2008). Acta Cryst. A64, 112-122.

Sheldrick, G. M. (2015). Acta Cryst. C71, 3-8.

Xia, J., Li, F., Yin, L., Yu, D. \& Wu, D. (2008). Acta Cryst. E64, 0632. 


\section{supporting information}

Acta Cryst. (2015). E71, o631-o632 [https://doi.org/10.1107/S2056989015014115]

\section{Crystal structure of cyclosulfamuron}

\section{Gihaeng Kang, Jineun Kim, Eunjin Kwon and Tae Ho Kim}

\section{S1. Comment}

Cyclosulfamuron [systematic name: 1-[2-(cyclopropylcarbonyl)anilinosulfonyl]-3-(4,6-dimethoxypyrimidin-2-yl)urea] is a pyrimidinylsulfonylurea herbicide and has been widely used to control weeds because of their low toxicity to mammals and their high herbicidal activity (Sarıgül \& Inam, 2009). However, until now its crystal structure has not been reported. In the title compound (Fig. 1), the dihedral angles between the mean planes of the central phenyl ring and the cyclopropyl and pyrimidinyl rings are 75.32 (9) and $88.79(4)^{\circ}$, respectively. All bond lengths and bond angles are normal and comparable to those observed in a similar crystal structure (Xia et al., 2008).

In the crystal structure (Fig. 2), $\mathrm{N}-\mathrm{H} \cdots \mathrm{O}$ and $\mathrm{C}-\mathrm{H} \cdots \mathrm{O}$ hydrogen bonds are observed (Table 1). In addition, weak intermolecular $C g 1 \cdots C g 1^{\mathrm{v}}$ and $C g 1 \cdots C g 2^{\text {vi }}(C g 1$ and $C g 2$ are the centroids of the $\mathrm{N} 4-\mathrm{C} 12-\mathrm{N} 5-\mathrm{C} 15-\mathrm{C} 14-\mathrm{C} 13$ and C5-C10 rings, respectively) interactions are present [for symmetry codes: (v), $-x+2,-y,-z+1,(\mathrm{vi}), x+1 / 2,-y+1 / 2, z+$ $1 / 2$ ]. A three-dimensional network is formed by the hydrogen bonds and $\pi-\pi$ interactions.

\section{S2. Experimental}

The title compound was purchased from the Dr. Ehrenstorfer $\mathrm{GmbH}$ Company. Slow evaporation of a solution in $\mathrm{CH}_{2} \mathrm{Cl}_{2}$ gave single crystals suitable for X-ray analysis.

\section{S3. Refinement}

All H-atoms were positioned geometrically and refined using a riding model with $\mathrm{d}(\mathrm{N}-\mathrm{H})=0.88 \AA, U_{\text {iso }}=1.2 U_{\text {eq }}(\mathrm{C})$ for $\mathrm{N}-\mathrm{H}$ group, $\mathrm{d}(\mathrm{C}-\mathrm{H})=1.00 \AA, U_{\text {iso }}=1.2 U_{\text {eq }}(\mathrm{C})$ for $\mathrm{C} s p^{3}-\mathrm{H}$. d $(\mathrm{C}-\mathrm{H})=0.98 \AA, U_{\text {iso }}=1.5 U_{\text {eq }}(\mathrm{C})$ for methyl group, $\mathrm{d}(\mathrm{C}$ $-\mathrm{H})=0.99 \AA, U_{\text {iso }}=1.2 U_{\text {eq }}(\mathrm{C})$ for $\mathrm{CH}_{2}$ group, $\mathrm{d}(\mathrm{C}-\mathrm{H})=0.95 \AA, U_{\text {iso }}=1.2 U_{\text {eq }}(\mathrm{C})$ for aromatic $\mathrm{C}-\mathrm{H}$. 


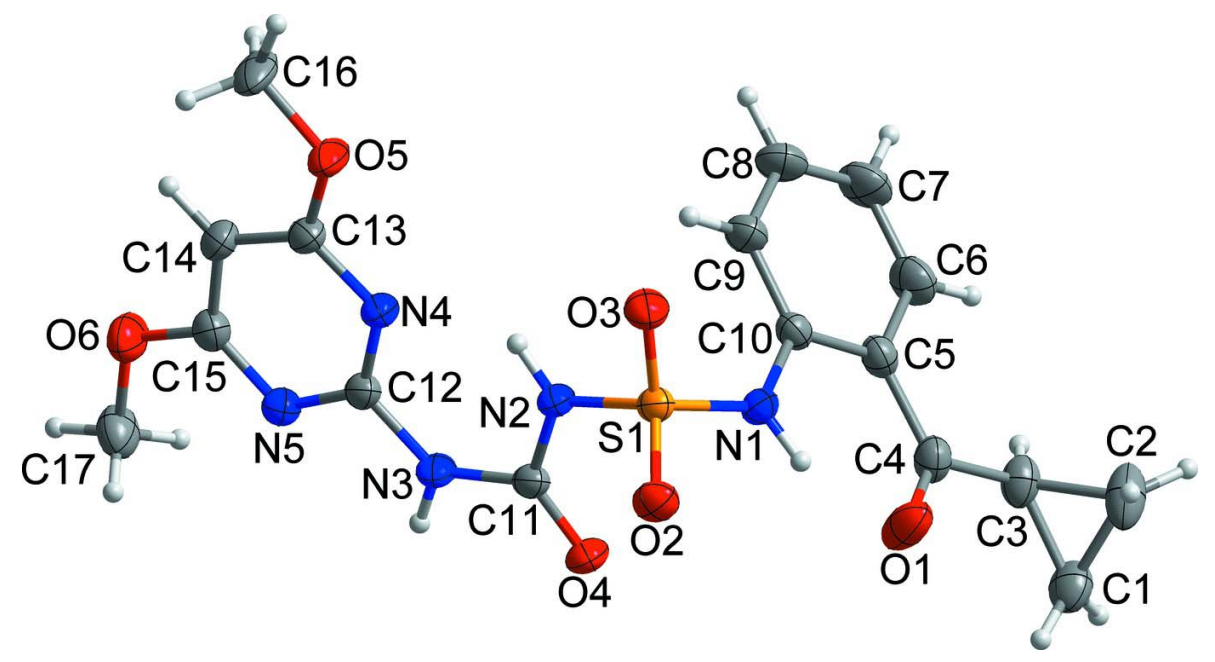

Figure 1

The asymmetric unit of the title compound with the atom numbering scheme. Displacement ellipsoids are drawn at the $50 \%$ probability level. $\mathrm{H}$ atoms are shown as small spheres of arbitrary radius.

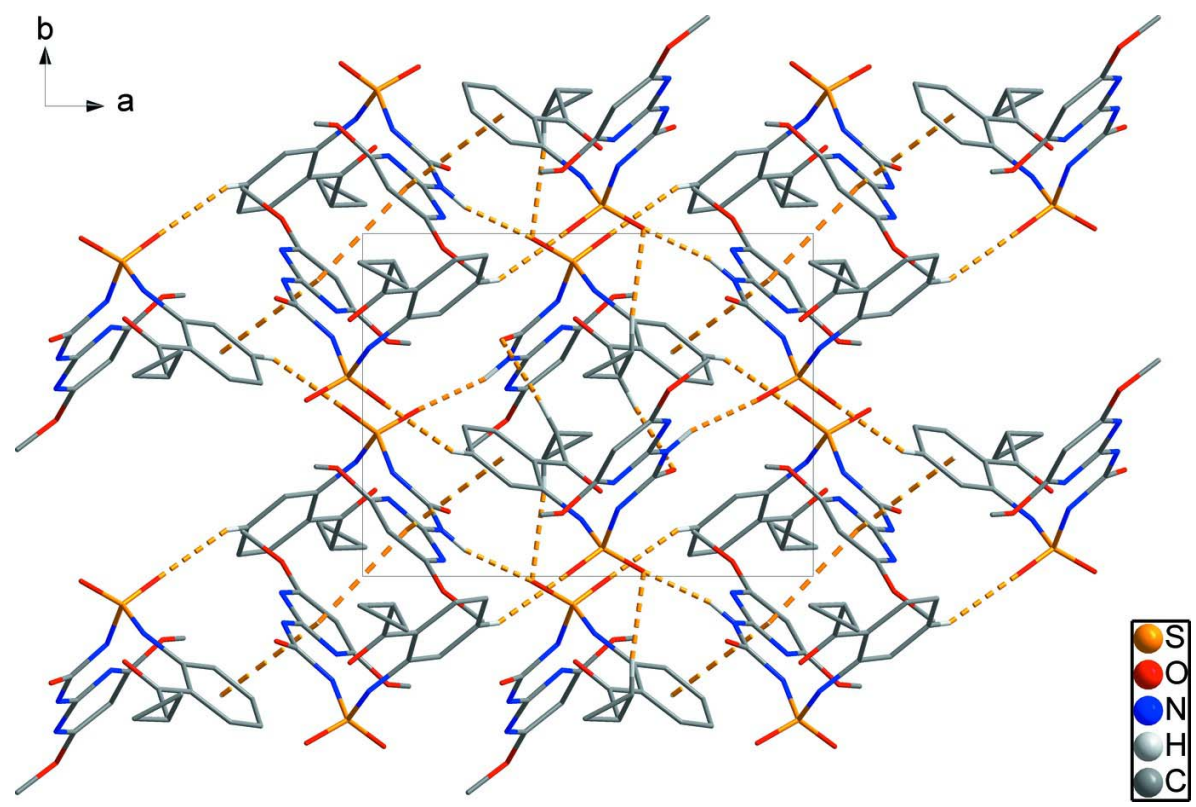

Figure 2

Crystal packing viewed along the $c$ axis. The intermolecular interactions are shown as dashed lines.

1-\{[2-(Cyclopropylcarbonyl)anilino]sulfonyl\}-3-(4,6-dimethoxypyrimidin-2-yl)urea

Crystal data

$\mathrm{C}_{17} \mathrm{H}_{19} \mathrm{~N}_{5} \mathrm{O}_{6} \mathrm{~S}$

$M_{r}=421.43$

Monoclinic, $P 2{ }_{1} / n$

$a=12.7019(4) \AA$

$b=9.6216(3) \AA$

$c=15.6213(5) \AA$

$\beta=93.6194(12)^{\circ}$

$$
\begin{aligned}
& V=1905.31(10) \AA^{3} \\
& Z=4 \\
& F(000)=880 \\
& D_{\mathrm{x}}=1.469 \mathrm{Mg} \mathrm{m}^{-3} \\
& \text { Mo } K \alpha \text { radiation, } \lambda=0.71073 \AA \\
& \text { Cell parameters from } 6977 \text { reflections } \\
& \theta=2.6-27.4^{\circ}
\end{aligned}
$$


$\begin{aligned} \mu & =0.22 \mathrm{~mm}^{-1} \\ T & =173 \mathrm{~K}\end{aligned}$

Data collection

Bruker APEXII CCD diffractometer

$\varphi$ and $\omega$ scans

Absorption correction: multi-scan

(SADABS; Bruker, 2013)

$T_{\min }=0.934, T_{\max }=0.952$

17544 measured reflections

\section{Refinement}

Refinement on $F^{2}$

Least-squares matrix: full

$R\left[F^{2}>2 \sigma\left(F^{2}\right)\right]=0.039$

$w R\left(F^{2}\right)=0.113$

$S=1.05$

4365 reflections

264 parameters

0 restraints
Block, colourless

$0.32 \times 0.27 \times 0.23 \mathrm{~mm}$

4365 independent reflections

3688 reflections with $I>2 \sigma(I)$

$R_{\text {int }}=0.030$

$\theta_{\text {max }}=27.5^{\circ}, \theta_{\text {min }}=2.0^{\circ}$

$h=-16 \rightarrow 15$

$k=-12 \rightarrow 12$

$l=-20 \rightarrow 20$

Hydrogen site location: inferred from neighbouring sites

$\mathrm{H}$-atom parameters constrained

$w=1 /\left[\sigma^{2}\left(F_{\mathrm{o}}^{2}\right)+(0.0579 P)^{2}+0.7248 P\right]$

where $P=\left(F_{\mathrm{o}}^{2}+2 F_{\mathrm{c}}{ }^{2}\right) / 3$

$(\Delta / \sigma)_{\max }=0.001$

$\Delta \rho_{\max }=0.22 \mathrm{e}^{-3}$

$\Delta \rho_{\min }=-0.49$ e $\AA^{-3}$

Special details

Geometry. All e.s.d.'s (except the e.s.d. in the dihedral angle between two 1.s. planes) are estimated using the full covariance matrix. The cell e.s.d.'s are taken into account individually in the estimation of e.s.d.'s in distances, angles and torsion angles; correlations between e.s.d.'s in cell parameters are only used when they are defined by crystal symmetry. An approximate (isotropic) treatment of cell e.s.d.'s is used for estimating e.s.d.'s involving l.s. planes.

Fractional atomic coordinates and isotropic or equivalent isotropic displacement parameters $\left(\AA^{2}\right)$

\begin{tabular}{lllll}
\hline & $x$ & $y$ & $z$ & $U_{\text {iso }} * / U_{\text {eq }}$ \\
\hline S1 & $1.03274(3)$ & $0.41778(4)$ & $0.19915(2)$ & $0.02564(12)$ \\
O1 & $1.02757(11)$ & $0.25129(16)$ & $-0.03440(8)$ & $0.0524(4)$ \\
O2 & $1.12410(9)$ & $0.48608(12)$ & $0.17159(7)$ & $0.0340(3)$ \\
O3 & $0.95254(9)$ & $0.49585(12)$ & $0.23699(7)$ & $0.0343(3)$ \\
O4 & $1.19071(9)$ & $0.18921(12)$ & $0.19997(7)$ & $0.0335(3)$ \\
O5 & $0.94327(9)$ & $0.31556(13)$ & $0.52112(7)$ & $0.0386(3)$ \\
O6 & $1.17908(10)$ & $-0.04334(14)$ & $0.61128(7)$ & $0.0429(3)$ \\
N1 & $0.98567(10)$ & $0.33405(14)$ & $0.11638(8)$ & $0.0290(3)$ \\
H1N & 1.0187 & 0.3456 & 0.0690 & $0.035^{*}$ \\
N2 & $1.06665(10)$ & $0.30293(14)$ & $0.27345(8)$ & $0.0281(3)$ \\
H2N & 1.0317 & 0.3014 & 0.3203 & $0.034^{*}$ \\
N3 & $1.17533(10)$ & $0.13526(15)$ & $0.33969(8)$ & $0.0303(3)$ \\
H3N & 1.2279 & 0.0767 & 0.3351 & $0.036^{*}$ \\
N4 & $1.05707(10)$ & $0.22678(14)$ & $0.43352(8)$ & $0.0284(3)$ \\
N5 & $1.17993(10)$ & $0.04736(14)$ & $0.47520(8)$ & $0.0302(3)$ \\
C1 & $1.00581(16)$ & $0.0853(2)$ & $-0.18258(11)$ & $0.0418(4)$ \\
H1A & 1.0695 & 0.1442 & -0.1740 & $0.050^{*}$ \\
H1B & 1.0162 & -0.0019 & -0.2147 & $0.050^{*}$ \\
C2 & $0.90252(18)$ & $0.1560(2)$ & $-0.19567(11)$ & $0.0492(5)$ \\
H2A & 0.8486 & 0.1127 & -0.2357 & $0.059^{*}$
\end{tabular}




$\begin{array}{lllll}\text { H2B } & 0.9019 & 0.2589 & -0.1950 & 0.059^{*} \\ \text { C3 } & 0.93094(14) & 0.08205(17) & -0.11251(10) & 0.0354(4) \\ \text { H3 } & 0.8943 & -0.0082 & -0.1031 & 0.042^{*} \\ \text { C4 } & 0.95231(14) & 0.17112(17) & -0.03618(10) & 0.0334(4) \\ \text { C5 } & 0.88087(12) & 0.16383(17) & 0.03524(10) & 0.0301(3) \\ \text { C6 } & 0.79319(14) & 0.0755(2) & 0.02989(12) & 0.0415(4) \\ \text { H6 } & 0.7797 & 0.0215 & -0.0206 & 0.050^{*} \\ \text { C7 } & 0.72575(15) & 0.0639(2) & 0.09523(13) & 0.0462(5) \\ \text { H7 } & 0.6672 & 0.0023 & 0.0901 & 0.055^{*} \\ \text { C8 } & 0.74454(14) & 0.1432(2) & 0.16818(12) & 0.0432(4) \\ \text { H8 } & 0.6987 & 0.1354 & 0.2137 & 0.052^{*} \\ \text { C9 } & 0.82919(13) & 0.23370(19) & 0.17609(10) & 0.0347(4) \\ \text { H9 } & 0.8403 & 0.2886 & 0.2264 & 0.042^{*} \\ \text { C10 } & 0.89805(12) & 0.24451(16) & 0.11062(9) & 0.0267(3) \\ \text { C11 } & 1.14737(12) & 0.20858(16) & 0.26595(9) & 0.0267(3) \\ \text { C12 } & 1.13446(12) & 0.13831(16) & 0.42007(9) & 0.0272(3) \\ \text { C13 } & 1.01967(12) & 0.22237(17) & 0.51212(9) & 0.0294(3) \\ \text { C14 } & 1.05796(13) & 0.13144(18) & 0.57482(9) & 0.0325(4) \\ \text { H14 } & 1.0303 & 0.1274 & 0.6299 & 0.039^{*} \\ \text { C15 } & 1.13996(13) & 0.04587(18) & 0.55188(10) & 0.0314(3) \\ \text { C16 } & 0.89737(16) & 0.3201(2) & 0.60284(11) & 0.0514(5) \\ \text { H16A } & 0.8605 & 0.2326 & 0.6124 & 0.077^{*} \\ \text { H16B } & 0.8472 & 0.3974 & 0.6035 & 0.077^{*} \\ \text { H16C } & 0.9531 & 0.3336 & 0.6484 & 0.077^{*} \\ \text { C17 } & 1.26553(16) & -0.1300(2) & 0.58797(12) & 0.0463(5) \\ \text { H17A } & 1.2435 & -0.1851 & 0.5372 & 0.069^{*} \\ \text { H17B } & 1.2862 & -0.1925 & 0.6357 & 0.069^{*} \\ \text { H17C } & 1.3256 & -0.0714 & 0.5752 & 0.069^{*}\end{array}$

Atomic displacement parameters $\left(\AA^{2}\right)$

\begin{tabular}{lllllll}
\hline & $U^{11}$ & $U^{22}$ & $U^{33}$ & $U^{12}$ & $U^{13}$ & $U^{23}$ \\
\hline $\mathrm{S} 1$ & $0.0234(2)$ & $0.0291(2)$ & $0.02454(19)$ & $-0.00228(14)$ & $0.00254(14)$ & $-0.00382(13)$ \\
$\mathrm{O} 1$ & $0.0535(9)$ & $0.0729(10)$ & $0.0323(6)$ & $-0.0295(7)$ & $0.0133(6)$ & $-0.0169(6)$ \\
$\mathrm{O} 2$ & $0.0313(6)$ & $0.0348(6)$ & $0.0362(6)$ & $-0.0109(5)$ & $0.0052(5)$ & $-0.0047(5)$ \\
$\mathrm{O} 3$ & $0.0321(6)$ & $0.0368(6)$ & $0.0343(6)$ & $0.0076(5)$ & $0.0039(5)$ & $-0.0045(5)$ \\
$\mathrm{O} 4$ & $0.0305(6)$ & $0.0445(7)$ & $0.0263(5)$ & $0.0045(5)$ & $0.0096(4)$ & $-0.0049(5)$ \\
$\mathrm{O} 5$ & $0.0368(7)$ & $0.0544(8)$ & $0.0255(6)$ & $0.0162(6)$ & $0.0090(5)$ & $-0.0010(5)$ \\
$\mathrm{O} 6$ & $0.0459(8)$ & $0.0518(8)$ & $0.0309(6)$ & $0.0133(6)$ & $0.0007(5)$ & $0.0070(5)$ \\
$\mathrm{N} 1$ & $0.0263(7)$ & $0.0387(7)$ & $0.0222(6)$ & $-0.0083(6)$ & $0.0039(5)$ & $-0.0029(5)$ \\
$\mathrm{N} 2$ & $0.0243(7)$ & $0.0385(7)$ & $0.0221(6)$ & $0.0048(5)$ & $0.0055(5)$ & $-0.0010(5)$ \\
$\mathrm{N} 3$ & $0.0256(7)$ & $0.0394(7)$ & $0.0263(6)$ & $0.0096(6)$ & $0.0059(5)$ & $-0.0015(5)$ \\
$\mathrm{N} 4$ & $0.0253(7)$ & $0.0370(7)$ & $0.0232(6)$ & $0.0037(5)$ & $0.0039(5)$ & $-0.0023(5)$ \\
$\mathrm{N} 5$ & $0.0263(7)$ & $0.0368(7)$ & $0.0274(6)$ & $0.0026(6)$ & $0.0016(5)$ & $-0.0017(5)$ \\
$\mathrm{C} 1$ & $0.0512(11)$ & $0.0429(10)$ & $0.0312(8)$ & $0.0058(8)$ & $0.0010(8)$ & $-0.0081(7)$ \\
$\mathrm{C} 2$ & $0.0760(15)$ & $0.0388(10)$ & $0.0310(9)$ & $0.0193(10)$ & $-0.0112(9)$ & $-0.0048(7)$ \\
$\mathrm{C} 3$ & $0.0467(10)$ & $0.0305(8)$ & $0.0283(8)$ & $0.0018(7)$ & $-0.0033(7)$ & $-0.0030(6)$ \\
$\mathrm{C} 4$ & $0.0368(9)$ & $0.0366(9)$ & $0.0262(7)$ & $-0.0024(7)$ & $-0.0031(6)$ & $-0.0015(6)$
\end{tabular}




\begin{tabular}{lllllll} 
C5 & $0.0270(8)$ & $0.0333(8)$ & $0.0292(7)$ & $-0.0029(6)$ & $-0.0049(6)$ & $0.0009(6)$ \\
C6 & $0.0348(10)$ & $0.0436(10)$ & $0.0451(10)$ & $-0.0099(8)$ & $-0.0048(8)$ & $-0.0083(8)$ \\
C7 & $0.0301(9)$ & $0.0511(11)$ & $0.0571(11)$ & $-0.0163(8)$ & $-0.0002(8)$ & $-0.0050(9)$ \\
C8 & $0.0282(9)$ & $0.0552(11)$ & $0.0471(10)$ & $-0.0093(8)$ & $0.0086(7)$ & $0.0018(9)$ \\
C9 & $0.0255(8)$ & $0.0450(10)$ & $0.0338(8)$ & $-0.0062(7)$ & $0.0027(6)$ & $-0.0025(7)$ \\
C10 & $0.0220(7)$ & $0.0304(8)$ & $0.0272(7)$ & $-0.0022(6)$ & $-0.0019(6)$ & $0.0015(6)$ \\
C11 & $0.0209(7)$ & $0.0342(8)$ & $0.0252(7)$ & $-0.0015(6)$ & $0.0030(5)$ & $-0.0046(6)$ \\
C12 & $0.0234(7)$ & $0.0346(8)$ & $0.0238(7)$ & $-0.0015(6)$ & $0.0017(6)$ & $-0.0037(6)$ \\
C13 & $0.0260(8)$ & $0.0379(8)$ & $0.0244(7)$ & $0.0015(6)$ & $0.0033(6)$ & $-0.0057(6)$ \\
C14 & $0.0328(9)$ & $0.0429(9)$ & $0.0220(7)$ & $0.0020(7)$ & $0.0036(6)$ & $-0.0029(6)$ \\
C15 & $0.0287(8)$ & $0.0383(9)$ & $0.0270(7)$ & $-0.0005(7)$ & $-0.0013(6)$ & $-0.0006(6)$ \\
C16 & $0.0513(12)$ & $0.0780(15)$ & $0.0262(8)$ & $0.0277(11)$ & $0.0122(8)$ & $-0.0043(9)$ \\
C17 & $0.0476(11)$ & $0.0523(11)$ & $0.0379(9)$ & $0.0167(9)$ & $-0.0058(8)$ & $0.0016(8)$ \\
\hline
\end{tabular}

Geometric parameters $\left(\AA,{ }^{\circ}\right)$

\begin{tabular}{|c|c|c|c|}
\hline $\mathrm{S} 1-\mathrm{O} 2$ & $1.4237(11)$ & $\mathrm{C} 2-\mathrm{C} 3$ & $1.505(2)$ \\
\hline $\mathrm{S} 1-\mathrm{O} 3$ & $1.4238(11)$ & $\mathrm{C} 2-\mathrm{H} 2 \mathrm{~A}$ & 0.9900 \\
\hline $\mathrm{S} 1-\mathrm{N} 1$ & $1.6065(12)$ & $\mathrm{C} 2-\mathrm{H} 2 \mathrm{~B}$ & 0.9900 \\
\hline $\mathrm{S} 1-\mathrm{N} 2$ & $1.6402(13)$ & $\mathrm{C} 3-\mathrm{C} 4$ & $1.480(2)$ \\
\hline $\mathrm{O} 1-\mathrm{C} 4$ & $1.227(2)$ & $\mathrm{C} 3-\mathrm{H} 3$ & 1.0000 \\
\hline $\mathrm{O} 4-\mathrm{C} 11$ & $1.2132(17)$ & $\mathrm{C} 4-\mathrm{C} 5$ & $1.484(2)$ \\
\hline $\mathrm{O} 5-\mathrm{C} 13$ & $1.3351(19)$ & $\mathrm{C} 5-\mathrm{C} 6$ & $1.399(2)$ \\
\hline $\mathrm{O} 5-\mathrm{C} 16$ & $1.4372(19)$ & $\mathrm{C} 5-\mathrm{C} 10$ & $1.416(2)$ \\
\hline $\mathrm{O} 6-\mathrm{C} 15$ & $1.3366(19)$ & $\mathrm{C} 6-\mathrm{C} 7$ & $1.378(3)$ \\
\hline $\mathrm{O} 6-\mathrm{C} 17$ & $1.444(2)$ & $\mathrm{C} 6-\mathrm{H} 6$ & 0.9500 \\
\hline $\mathrm{N} 1-\mathrm{C} 10$ & $1.4058(19)$ & $\mathrm{C} 7-\mathrm{C} 8$ & $1.379(3)$ \\
\hline $\mathrm{N} 1-\mathrm{H} 1 \mathrm{~N}$ & 0.8800 & $\mathrm{C} 7-\mathrm{H} 7$ & 0.9500 \\
\hline $\mathrm{N} 2-\mathrm{C} 11$ & $1.3799(19)$ & $\mathrm{C} 8-\mathrm{C} 9$ & $1.383(2)$ \\
\hline $\mathrm{N} 2-\mathrm{H} 2 \mathrm{~N}$ & 0.8800 & $\mathrm{C} 8-\mathrm{H} 8$ & 0.9500 \\
\hline $\mathrm{N} 3-\mathrm{C} 11$ & $1.378(2)$ & $\mathrm{C} 9-\mathrm{C} 10$ & $1.391(2)$ \\
\hline $\mathrm{N} 3-\mathrm{C} 12$ & $1.3890(18)$ & $\mathrm{C} 9-\mathrm{H} 9$ & 0.9500 \\
\hline $\mathrm{N} 3-\mathrm{H} 3 \mathrm{~N}$ & 0.8800 & $\mathrm{C} 13-\mathrm{C} 14$ & $1.379(2)$ \\
\hline $\mathrm{N} 4-\mathrm{C} 12$ & $1.327(2)$ & $\mathrm{C} 14-\mathrm{C} 15$ & $1.392(2)$ \\
\hline $\mathrm{N} 4-\mathrm{C} 13$ & $1.3449(19)$ & $\mathrm{C} 14-\mathrm{H} 14$ & 0.9500 \\
\hline $\mathrm{N} 5-\mathrm{C} 15$ & $1.3303(19)$ & $\mathrm{C} 16-\mathrm{H} 16 \mathrm{~A}$ & 0.9800 \\
\hline N5-C12 & $1.333(2)$ & C16-H16B & 0.9800 \\
\hline $\mathrm{C} 1-\mathrm{C} 2$ & $1.480(3)$ & $\mathrm{C} 16-\mathrm{H} 16 \mathrm{C}$ & 0.9800 \\
\hline $\mathrm{C} 1-\mathrm{C} 3$ & $1.495(2)$ & C17-H17A & 0.9800 \\
\hline $\mathrm{C} 1-\mathrm{H} 1 \mathrm{~A}$ & 0.9900 & C17-H17B & 0.9800 \\
\hline $\mathrm{C} 1-\mathrm{H} 1 \mathrm{~B}$ & 0.9900 & $\mathrm{C} 17-\mathrm{H} 17 \mathrm{C}$ & 0.9800 \\
\hline $\mathrm{O} 2-\mathrm{S} 1-\mathrm{O} 3$ & $120.02(7)$ & $\mathrm{C} 7-\mathrm{C} 6-\mathrm{C} 5$ & $122.31(17)$ \\
\hline $\mathrm{O} 2-\mathrm{S} 1-\mathrm{N} 1$ & $104.90(7)$ & $\mathrm{C} 7-\mathrm{C} 6-\mathrm{H} 6$ & 118.8 \\
\hline $\mathrm{O} 3-\mathrm{S} 1-\mathrm{N} 1$ & $111.04(7)$ & $\mathrm{C} 5-\mathrm{C} 6-\mathrm{H} 6$ & 118.8 \\
\hline $\mathrm{O} 2-\mathrm{S} 1-\mathrm{N} 2$ & $110.02(7)$ & $\mathrm{C} 6-\mathrm{C} 7-\mathrm{C} 8$ & $118.96(17)$ \\
\hline $\mathrm{O} 3-\mathrm{S} 1-\mathrm{N} 2$ & $102.97(7)$ & $\mathrm{C} 6-\mathrm{C} 7-\mathrm{H} 7$ & 120.5 \\
\hline $\mathrm{N} 1-\mathrm{S} 1-\mathrm{N} 2$ & $107.42(7)$ & $\mathrm{C} 8-\mathrm{C} 7-\mathrm{H} 7$ & 120.5 \\
\hline
\end{tabular}




\begin{tabular}{|c|c|c|c|}
\hline $\mathrm{C} 13-\mathrm{O} 5-\mathrm{C} 16$ & $116.90(13)$ & $\mathrm{C} 7-\mathrm{C} 8-\mathrm{C} 9$ & $120.99(17)$ \\
\hline $\mathrm{C} 15-\mathrm{O} 6-\mathrm{C} 17$ & $116.82(13)$ & $\mathrm{C} 7-\mathrm{C} 8-\mathrm{H} 8$ & 119.5 \\
\hline $\mathrm{C} 10-\mathrm{N} 1-\mathrm{S} 1$ & $127.72(10)$ & $\mathrm{C} 9-\mathrm{C} 8-\mathrm{H} 8$ & 119.5 \\
\hline $\mathrm{C} 10-\mathrm{N} 1-\mathrm{H} 1 \mathrm{~N}$ & 116.1 & $\mathrm{C} 8-\mathrm{C} 9-\mathrm{C} 10$ & $120.18(16)$ \\
\hline $\mathrm{S} 1-\mathrm{N} 1-\mathrm{H} 1 \mathrm{~N}$ & 116.1 & $\mathrm{C} 8-\mathrm{C} 9-\mathrm{H} 9$ & 119.9 \\
\hline $\mathrm{C} 11-\mathrm{N} 2-\mathrm{S} 1$ & $123.13(10)$ & $\mathrm{C} 10-\mathrm{C} 9-\mathrm{H} 9$ & 119.9 \\
\hline $\mathrm{C} 11-\mathrm{N} 2-\mathrm{H} 2 \mathrm{~N}$ & 118.4 & $\mathrm{C} 9-\mathrm{C} 10-\mathrm{N} 1$ & $122.06(14)$ \\
\hline $\mathrm{S} 1-\mathrm{N} 2-\mathrm{H} 2 \mathrm{~N}$ & 118.4 & $\mathrm{C} 9-\mathrm{C} 10-\mathrm{C} 5$ & $119.97(14)$ \\
\hline $\mathrm{C} 11-\mathrm{N} 3-\mathrm{C} 12$ & $130.81(13)$ & $\mathrm{N} 1-\mathrm{C} 10-\mathrm{C} 5$ & $117.98(13)$ \\
\hline $\mathrm{C} 11-\mathrm{N} 3-\mathrm{H} 3 \mathrm{~N}$ & 114.6 & $\mathrm{O} 4-\mathrm{C} 11-\mathrm{N} 3$ & $121.65(14)$ \\
\hline $\mathrm{C} 12-\mathrm{N} 3-\mathrm{H} 3 \mathrm{~N}$ & 114.6 & $\mathrm{O} 4-\mathrm{C} 11-\mathrm{N} 2$ & $123.54(14)$ \\
\hline $\mathrm{C} 12-\mathrm{N} 4-\mathrm{C} 13$ & $115.60(13)$ & $\mathrm{N} 3-\mathrm{C} 11-\mathrm{N} 2$ & $114.81(12)$ \\
\hline $\mathrm{C} 15-\mathrm{N} 5-\mathrm{C} 12$ & $114.47(13)$ & $\mathrm{N} 4-\mathrm{C} 12-\mathrm{N} 5$ & $127.86(14)$ \\
\hline $\mathrm{C} 2-\mathrm{C} 1-\mathrm{C} 3$ & $60.76(12)$ & $\mathrm{N} 4-\mathrm{C} 12-\mathrm{N} 3$ & $118.65(14)$ \\
\hline $\mathrm{C} 2-\mathrm{C} 1-\mathrm{H} 1 \mathrm{~A}$ & 117.7 & $\mathrm{~N} 5-\mathrm{C} 12-\mathrm{N} 3$ & $113.49(13)$ \\
\hline $\mathrm{C} 3-\mathrm{C} 1-\mathrm{H} 1 \mathrm{~A}$ & 117.7 & $\mathrm{O} 5-\mathrm{C} 13-\mathrm{N} 4$ & $112.08(13)$ \\
\hline $\mathrm{C} 2-\mathrm{C} 1-\mathrm{H} 1 \mathrm{~B}$ & 117.7 & $\mathrm{O} 5-\mathrm{C} 13-\mathrm{C} 14$ & $125.27(14)$ \\
\hline $\mathrm{C} 3-\mathrm{C} 1-\mathrm{H} 1 \mathrm{~B}$ & 117.7 & $\mathrm{~N} 4-\mathrm{C} 13-\mathrm{C} 14$ & $122.65(14)$ \\
\hline $\mathrm{H} 1 \mathrm{~A}-\mathrm{C} 1-\mathrm{H} 1 \mathrm{~B}$ & 114.8 & $\mathrm{C} 13-\mathrm{C} 14-\mathrm{C} 15$ & $115.36(14)$ \\
\hline $\mathrm{C} 1-\mathrm{C} 2-\mathrm{C} 3$ & $60.10(12)$ & $\mathrm{C} 13-\mathrm{C} 14-\mathrm{H} 14$ & 122.3 \\
\hline $\mathrm{C} 1-\mathrm{C} 2-\mathrm{H} 2 \mathrm{~A}$ & 117.8 & $\mathrm{C} 15-\mathrm{C} 14-\mathrm{H} 14$ & 122.3 \\
\hline $\mathrm{C} 3-\mathrm{C} 2-\mathrm{H} 2 \mathrm{~A}$ & 117.8 & $\mathrm{~N} 5-\mathrm{C} 15-\mathrm{O} 6$ & $119.06(15)$ \\
\hline $\mathrm{C} 1-\mathrm{C} 2-\mathrm{H} 2 \mathrm{~B}$ & 117.8 & $\mathrm{~N} 5-\mathrm{C} 15-\mathrm{C} 14$ & $124.04(15)$ \\
\hline $\mathrm{C} 3-\mathrm{C} 2-\mathrm{H} 2 \mathrm{~B}$ & 117.8 & $\mathrm{O} 6-\mathrm{C} 15-\mathrm{C} 14$ & $116.89(14)$ \\
\hline $\mathrm{H} 2 \mathrm{~A}-\mathrm{C} 2-\mathrm{H} 2 \mathrm{~B}$ & 114.9 & $\mathrm{O} 5-\mathrm{C} 16-\mathrm{H} 16 \mathrm{~A}$ & 109.5 \\
\hline $\mathrm{C} 4-\mathrm{C} 3-\mathrm{C} 1$ & $119.02(16)$ & $\mathrm{O} 5-\mathrm{C} 16-\mathrm{H} 16 \mathrm{~B}$ & 109.5 \\
\hline $\mathrm{C} 4-\mathrm{C} 3-\mathrm{C} 2$ & $116.34(14)$ & $\mathrm{H} 16 \mathrm{~A}-\mathrm{C} 16-\mathrm{H} 16 \mathrm{~B}$ & 109.5 \\
\hline $\mathrm{C} 1-\mathrm{C} 3-\mathrm{C} 2$ & $59.14(12)$ & $\mathrm{O} 5-\mathrm{C} 16-\mathrm{H} 16 \mathrm{C}$ & 109.5 \\
\hline $\mathrm{C} 4-\mathrm{C} 3-\mathrm{H} 3$ & 116.6 & $\mathrm{H} 16 \mathrm{~A}-\mathrm{C} 16-\mathrm{H} 16 \mathrm{C}$ & 109.5 \\
\hline $\mathrm{C} 1-\mathrm{C} 3-\mathrm{H} 3$ & 116.6 & $\mathrm{H} 16 \mathrm{~B}-\mathrm{C} 16-\mathrm{H} 16 \mathrm{C}$ & 109.5 \\
\hline $\mathrm{C} 2-\mathrm{C} 3-\mathrm{H} 3$ & 116.6 & $\mathrm{O} 6-\mathrm{C} 17-\mathrm{H} 17 \mathrm{~A}$ & 109.5 \\
\hline $\mathrm{O} 1-\mathrm{C} 4-\mathrm{C} 3$ & $119.04(15)$ & $\mathrm{O} 6-\mathrm{C} 17-\mathrm{H} 17 \mathrm{~B}$ & 109.5 \\
\hline $\mathrm{O} 1-\mathrm{C} 4-\mathrm{C} 5$ & $121.68(14)$ & $\mathrm{H} 17 \mathrm{~A}-\mathrm{C} 17-\mathrm{H} 17 \mathrm{~B}$ & 109.5 \\
\hline $\mathrm{C} 3-\mathrm{C} 4-\mathrm{C} 5$ & $119.26(15)$ & $\mathrm{O} 6-\mathrm{C} 17-\mathrm{H} 17 \mathrm{C}$ & 109.5 \\
\hline $\mathrm{C} 6-\mathrm{C} 5-\mathrm{C} 10$ & $117.59(15)$ & $\mathrm{H} 17 \mathrm{~A}-\mathrm{C} 17-\mathrm{H} 17 \mathrm{C}$ & 109.5 \\
\hline $\mathrm{C} 6-\mathrm{C} 5-\mathrm{C} 4$ & $120.40(15)$ & $\mathrm{H} 17 \mathrm{~B}-\mathrm{C} 17-\mathrm{H} 17 \mathrm{C}$ & 109.5 \\
\hline $\mathrm{C} 10-\mathrm{C} 5-\mathrm{C} 4$ & $122.01(14)$ & & \\
\hline $\mathrm{O} 2-\mathrm{S} 1-\mathrm{N} 1-\mathrm{C} 10$ & $176.89(13)$ & $\mathrm{C} 4-\mathrm{C} 5-\mathrm{C} 10-\mathrm{C} 9$ & $-179.69(15)$ \\
\hline $\mathrm{O} 3-\mathrm{S} 1-\mathrm{N} 1-\mathrm{C} 10$ & $-52.05(15)$ & $\mathrm{C} 6-\mathrm{C} 5-\mathrm{C} 10-\mathrm{N} 1$ & $-179.72(15)$ \\
\hline $\mathrm{N} 2-\mathrm{S} 1-\mathrm{N} 1-\mathrm{C} 10$ & $59.84(15)$ & $\mathrm{C} 4-\mathrm{C} 5-\mathrm{C} 10-\mathrm{N} 1$ & $0.3(2)$ \\
\hline $\mathrm{O} 2-\mathrm{S} 1-\mathrm{N} 2-\mathrm{C} 11$ & $-47.34(14)$ & $\mathrm{C} 12-\mathrm{N} 3-\mathrm{C} 11-\mathrm{O} 4$ & $-177.90(15)$ \\
\hline $\mathrm{O} 3-\mathrm{S} 1-\mathrm{N} 2-\mathrm{C} 11$ & $-176.41(12)$ & $\mathrm{C} 12-\mathrm{N} 3-\mathrm{C} 11-\mathrm{N} 2$ & $1.5(2)$ \\
\hline $\mathrm{N} 1-\mathrm{S} 1-\mathrm{N} 2-\mathrm{C} 11$ & $66.31(13)$ & $\mathrm{S} 1-\mathrm{N} 2-\mathrm{C} 11-\mathrm{O} 4$ & $-10.3(2)$ \\
\hline $\mathrm{C} 2-\mathrm{C} 1-\mathrm{C} 3-\mathrm{C} 4$ & $-105.05(18)$ & $\mathrm{S} 1-\mathrm{N} 2-\mathrm{C} 11-\mathrm{N} 3$ & $170.31(11)$ \\
\hline $\mathrm{C} 1-\mathrm{C} 2-\mathrm{C} 3-\mathrm{C} 4$ & $109.56(18)$ & $\mathrm{C} 13-\mathrm{N} 4-\mathrm{C} 12-\mathrm{N} 5$ & $-0.8(2)$ \\
\hline $\mathrm{C} 1-\mathrm{C} 3-\mathrm{C} 4-\mathrm{O} 1$ & $4.1(2)$ & $\mathrm{C} 13-\mathrm{N} 4-\mathrm{C} 12-\mathrm{N} 3$ & $178.68(14)$ \\
\hline $\mathrm{C} 2-\mathrm{C} 3-\mathrm{C} 4-\mathrm{O} 1$ & $-63.6(2)$ & $\mathrm{C} 15-\mathrm{N} 5-\mathrm{C} 12-\mathrm{N} 4$ & $1.3(2)$ \\
\hline
\end{tabular}




$\begin{array}{llll}\mathrm{C} 1-\mathrm{C} 3-\mathrm{C} 4-\mathrm{C} 5 & -177.36(15) & \mathrm{C} 15-\mathrm{N} 5-\mathrm{C} 12-\mathrm{N} 3 & -178.23(14) \\ \mathrm{C} 2-\mathrm{C} 3-\mathrm{C} 4-\mathrm{C} 5 & 114.96(19) & \mathrm{C} 11-\mathrm{N} 3-\mathrm{C} 12-\mathrm{N} 4 & -2.0(2) \\ \mathrm{O} 1-\mathrm{C} 4-\mathrm{C} 5-\mathrm{C} 6 & 177.40(17) & \mathrm{C} 11-\mathrm{N} 3-\mathrm{C} 12-\mathrm{N} 5 & 177.54(15) \\ \mathrm{C} 3-\mathrm{C} 4-\mathrm{C} 5-\mathrm{C} 6 & -1.1(2) & \mathrm{C} 16-\mathrm{O} 5-\mathrm{C} 13-\mathrm{N} 4 & -179.99(16) \\ \mathrm{O} 1-\mathrm{C} 4-\mathrm{C} 5-\mathrm{C} 10 & -2.6(3) & \mathrm{C} 16-\mathrm{O} 5-\mathrm{C} 13-\mathrm{C} 14 & 179.06(13) \\ \mathrm{C} 3-\mathrm{C} 4-\mathrm{C} 5-\mathrm{C} 10 & 178.88(14) & \mathrm{C} 12-\mathrm{N} 4-\mathrm{C} 13-\mathrm{O} 5 & -0.6(2) \\ \mathrm{C} 10-\mathrm{C} 5-\mathrm{C} 6-\mathrm{C} 7 & -1.0(3) & \mathrm{C} 12-\mathrm{N} 4-\mathrm{C} 13-\mathrm{C} 14 & -178.31(15) \\ \mathrm{C} 4-\mathrm{C} 5-\mathrm{C} 6-\mathrm{C} 7 & 179.00(18) & \mathrm{O} 5-\mathrm{C} 13-\mathrm{C} 14-\mathrm{C} 15 & 1.3(2) \\ \mathrm{C} 5-\mathrm{C} 6-\mathrm{C} 7-\mathrm{C} 8 & 0.7(3) & \mathrm{C} 12-\mathrm{N} 5-\mathrm{C} 15-\mathrm{O} 6 & 178.96(14) \\ \mathrm{C} 6-\mathrm{C} 7-\mathrm{C} 8-\mathrm{C} 9 & 0.4(3) & \mathrm{C} 12-\mathrm{N} 5-\mathrm{C} 15-\mathrm{C} 14 & -0.4(2) \\ \mathrm{C} 7-\mathrm{C} 8-\mathrm{C} 9-\mathrm{C} 10 & -1.0(3) & \mathrm{C} 17-\mathrm{O} 6-\mathrm{C} 15-\mathrm{N} 5 & 1.8(2) \\ \mathrm{C} 8-\mathrm{C} 9-\mathrm{C} 10-\mathrm{N} 1 & -179.31(16) & \mathrm{C} 17-\mathrm{O} 6-\mathrm{C} 15-\mathrm{C} 14 & -178.78(15) \\ \mathrm{C} 8-\mathrm{C} 9-\mathrm{C} 10-\mathrm{C} 5 & 0.7(3) & \mathrm{C} 13-\mathrm{C} 14-\mathrm{C} 15-\mathrm{N} 5 & -0.8(2) \\ \mathrm{S} 1-\mathrm{N} 1-\mathrm{C} 10-\mathrm{C} 9 & 10.3(2) & \mathrm{C} 13-\mathrm{C} 14-\mathrm{C} 15-\mathrm{O} 6 & 179.85(15) \\ \mathrm{S} 1-\mathrm{N} 1-\mathrm{C} 10-\mathrm{C} 5 & -169.74(12) & & \end{array}$

Hydrogen-bond geometry $\left(\AA,{ }^{\circ}\right)$

\begin{tabular}{lllll}
\hline$D-\mathrm{H} \cdots A$ & $D-\mathrm{H}$ & $\mathrm{H} \cdots A$ & $D \cdots A$ & $D-\mathrm{H} \cdots A$ \\
\hline $\mathrm{N} 1-\mathrm{H} 1 N \cdots \mathrm{O} 1$ & 0.88 & 1.86 & $2.5736(18)$ & 137 \\
$\mathrm{~N} 2-\mathrm{H} 2 N \cdots \mathrm{N} 4$ & 0.88 & 1.92 & $2.6158(18)$ & 135 \\
$\mathrm{C} 9-\mathrm{H} 9 \cdots \mathrm{O} 3$ & 0.95 & 2.45 & $3.088(2)$ & 124 \\
$\mathrm{~N} 3-\mathrm{H} 3 N \cdots \mathrm{O} 2^{\mathrm{i}}$ & 0.88 & 2.08 & $2.9391(17)$ & 165 \\
$\mathrm{C} 2-\mathrm{H} 2 B \cdots \mathrm{O} 2^{\mathrm{ii}}$ & 0.99 & 2.51 & $3.483(2)$ & 169 \\
$\mathrm{C} 3-\mathrm{H} 3 \cdots \mathrm{O} 4{ }^{\mathrm{iii}}$ & 1.00 & 2.51 & $3.286(2)$ & 135 \\
$\mathrm{C} 8-\mathrm{H} 8 \cdots{ }^{\text {iv }}$ & 0.95 & 2.50 & $3.307(2)$ & 142
\end{tabular}

Symmetry codes: (i) $-x+5 / 2, y-1 / 2,-z+1 / 2$; (ii) $-x+2,-y+1,-z$; (iii) $-x+2,-y,-z$; (iv) $-x+3 / 2, y-1 / 2,-z+1 / 2$. 